\title{
Entretien avec Sophie Lévy et Veerle Thielemans (Terra Foundation, Paris)
}

Le 1/12/08 à Reid Hall, Paris.

\section{François Brunet et Géraldine Chouard}

\section{(2) OpenEdition}

\section{Journals}

\section{Édition électronique}

URL : https://journals.openedition.org/transatlantica/4315

DOI : 10.4000/transatlantica.4315

ISSN : 1765-2766

\section{Éditeur}

Association française d'Etudes Américaines (AFEA)

\section{Référence électronique}

François Brunet et Géraldine Chouard, «Entretien avec Sophie Lévy et Veerle Thielemans (Terra

Foundation, Paris) », Transatlantica [En ligne], 1 | 2009, mis en ligne le 14 septembre 2009, consulté le 18 septembre 2021. URL : http://journals.openedition.org/transatlantica/4315 ; DOI : https://doi.org/ 10.4000/transatlantica.4315

Ce document a été généré automatiquement le 18 septembre 2021.

\section{(c) (i) (9)}

Transatlantica - Revue d'études américaines est mise à disposition selon les termes de la licence Creative Commons Attribution - Pas d'Utilisation Commerciale - Pas de Modification 4.0 International. 


\section{Entretien avec Sophie Lévy et Veerle Thielemans (Terra Foundation, Paris)}

Le 1/12/08 à Reid Hall, Paris.

François Brunet et Géraldine Chouard

François Brunet (FB): Quel bilan tirez-vous de l'expérience du MAAG ? Quels sont vos regrets, vos satisfactions? Peut-on dire rétrospectivement que ce musée était destiné à prendre fin de cette façon, après seize ans d'existence ? Y a-t-il des choses qui auraient pu ou dû être faites autrement?

Les plus grandes satisfactions?

Sophie Lévy (SL): On parle bien du Musée, puisque la Terra Summer Residency va se poursuivre à Giverny. Certaines expositions. Surtout, la transformation totale de la composition du public. On est passé d'un public de groupes d'Américains qui sortaient de la maison de Monet - au départ ils représentaient plus de $2 / 3$ des visiteurs - à un public de $2 / 3$ à $3 / 4$ de Français, surtout individuels, qui avaient entendu parler du musée et venaient ou revenaient portés par une vraie curiosité. [La nationalité était demandée aux visiteurs à l'entrée.] Cette transformation s'est faite entre 1998 (date d'arrivée de Derrick Cartwright) et 2008, et elle était déjà très claire en 2006. Le 11 septembre a ici joué un rôle, mais cela n'a été qu'un facteur parmi d'autres. Globalement, la fréquentation a augmenté ; quand je suis arrivée, elle était de 65 à 70 000, mais en 2007 (ainsi qu'en 2004) on est monté à 100000 visiteurs. 2007 a été une année intéressante parce qu'il y avait un bon équilibre entre chaque saison, qui a permis d'exploiter les potentialités du moment : "Giverny colonie impressionniste" était parfaite comme exposition de printemps, "Visions de l'Ouest » était une bonne exposition d'automne, avec une couverture de presse plus forte et un public plus pointu. Cette évolution a correspondu aussi à un intérêt croissant du public français vis-à-vis des écoles étrangères, une évolution à laquelle nous avons participé. 
FB : Vous avez fait allusion au 11 septembre, pouvez-vous en dire plus?

SL : La fréquentation américaine a baissé à ce moment-là, mais elle a été alors totalement compensée par une augmentation des visiteurs français. C'est une fierté, car l'objectif de Daniel Terra et de la Fondation n'a jamais été de faire un musée d'art américain pour les Américains. Là était également le sens d'expositions comme "Paris, capitale de l'Amérique. L'avant-garde américaine à Paris, 1939-1945" ou "Edward Hopper: les années parisiennes, 1906-1910" qui ciblaient l'échange transatlantique et le dialogue avec l'art européen, ce qui a été clairement perçu par le public. Par ailleurs il y a eu une adhésion croissante du public français au lieu, à son architecture simple, intégrée au paysage, qui est une réussite.

Veerle Thielemans (VL) : Mais finalement, 15 ans, ce n'est pas beaucoup, c'est très peu.

FB : D'où la deuxième question : y a-t-il des regrets ? Déjà, la fermeture du musée est-elle en elle-même un motif de regret?

VT : Ça dépend à quel niveau on se situe. Si on regarde l'histoire des implantations d'institutions culturelles américaines en dehors des Etats-Unis, d'abord il y en a très peu. De plus, il est rare que ce genre de structure s'enracine, parce qu'il n'y avait pas de modèle d'un lieu culturel américain durable, surtout privé. Le modèle nomade est plus naturel.

Géraldine Chouard (GC) : Parce que ce genre de lieu ne s'intègre pas dans le cadre culturel français?

VT : Ce n'est pas tant le pays d'accueil qui joue que...

SL : ...la mentalité américaine dans son rapport avec l'étranger.

FB : C'est-à-dire?

VT: On ne trouve pas aux Etats-Unis cette idée de former des ambassades de la culture américaine, du moins des ambassades fixes.

SL : Ça a été l'une des difficultés au départ. D'ailleurs ce musée était, au début, totalement isolé du milieu des musées français et européens. Venant du monde des musées publics français, je savais en arrivant qu'une consigne avait été clairement donnée de ne pas prêter à ce musée privé (menaçant, américain, etc.) et de ne pas collaborer avec lui. Là aussi, un certain nombre de facteurs ont permis de transformer cette situation, et c'est une autre satisfaction.

Mais pour revenir aux regrets, il y a aussi tous les projets qu'on aurait pu faire, dont on rêvait encore. On avait encore faim de beaucoup de choses.

Qu'est-ce qu'on aurait dû faire différemment? Comme dit Veerle, le modèle des musées américains est dominé par le Board, et il faut le convaincre constamment de l'importance de ce que l'on fait. Cette médiation a sans doute été l'aspect le plus difficile - c'est un regret personnel - je ne trouvais pas toujours les mots, les arguments, pour convaincre le Board de l'importance stratégique de ce musée. Par ailleurs, la transformation du musée de Giverny entre dans une histoire plus large de désengagement de la Fondation vis-à-vis de la gestion directe d'un musée - une majorité de membres du Board préfère la très grande liberté d'action que permet le mécénat de projets culturels afin de mettre en œuvre efficacement la mission de la fondation, qui est, comme vous le savez de promouvoir de manière très large l'art américain historique. 
VT : Et qu'ils l'empêchent d'avoir la réactivité voulue, permettant de faire beaucoup de choses à la fois.

GC : Mais pourtant c'est un ancrage culturel, une vraie implantation.

SL : Oui, mais limités. Et quels que soient les efforts qu'aurait pu faire le MAAG, il n'aurait sans doute pas pu dépasser le seuil de 150000 visiteurs sur ses 7 mois d'ouverture. Il y a une limite naturelle, liée à la situation hors de Paris, difficile d'accès, et au fait que c'est un petit musée. On l'a vu lors de l'exposition Hopper, qui avait attiré beaucoup de gens : on a une limite de capacité d'accueil. Or la question, du point de vue d'une grande partie du Board est l'impact des actions menées.

FB : Peut-on préciser, justement, quel était au départ le but poursuivi par la Fondation avec ce musée? II ne s'agissait sûrement pas d'un simple retour financier sur investissement.

SL : Il y a eu plusieurs étapes. Daniel Terra, d'abord, avait un certain nombre de tableaux peints à Giverny et il voulait créer un écrin pour ses tableaux, pour se faire plaisir. Pour lui, montrer les tableaux dans leur contexte de production était un réel avantage. Passons sur la période de transition (avant 1998). Quand le Board a recruté D. Cartwright, un historien de l'art, ils avaient un projet beaucoup plus ambitieux : ils voulaient refaire une sorte de centre culturel américain, d'expositions, de résidence, de création, de pensée, et cela a été un moment d'ambition très fort - une deuxième étape. Comme je l'ai indiqué précédemment, la décision par le Board de fermer le Terra Museum de Chicago s'est ensuite étendue à la question plus générale du financement direct des musées - qui immobilisent des crédits importants qui pourraient être distribués sur de multiples projets, dans de nombreux lieux différents.

VT : Je voudrais revenir sur la première étape. Il y avait chez Daniel Terra un aspect visionnaire, cette idée un peu folle de faire vivre la colonie d'artistes à Giverny, un côté utopique qui ne peut pas se réduire au seul but d'exposer sa collection. Cela a inspiré la deuxième phase, même si ce n'était pas explicite ; il y a eu par exemple des expériences sur la résidence d'été dans ce but.

SL : Et les maisons du village [dont la Fondation est aujourd'hui propriétaire] ont été achetées dans cette optique-là. Il y avait aussi, déjà, l'idée d'avoir une bibliothèque.

FB : Il y avait quand même un maillon faible dans ce projet : la localisation, qui empêchait a priori d'envisager une mission éducative de grande ampleur.

VT : C'est vrai qu'il y avait à la fois la distance de Paris, le voisinage avec une autre institution culturelle qui n'a rien à voir [la maison de Monet], et la petite taille, le côté rural.... Les premières formes d'activités pédagogiques que nous avons tentées (conférences le dimanche, histoire de l'art, etc.) ont eu du mal à trouver leur public, parce que le vivier local était trop limité.

GC : Et les résidences d'été, ça a commencé tout de suite?

VT : Il y a eu en 1996, je crois, un essai avec une étudiante employée à mi-temps pour accueillir des étudiants américains, des artistes surtout. Ça a échoué parce qu'il n'y avait pas de cadre. La TSR telle qu'on la connaît a commencé en 2001, c'est-à-dire qu'elle va avoir neuf ans, ce qui, sur une histoire d'une quinzaine d'années, n'est pas mal et renforce le côté " visionnaire ". Je voudrais dire ici plusieurs choses. D'abord, que la mission éducative a fait partie du rêve de Daniel Terra et a été poursuivie par la fondation dès le début du musée. Le fait d'associer un programme éducatif 
ambitieux tel que la TSR (international, de niveau universitaire) aux activités pédagogiques plus classiques d'un musée atteste de cela. A maintes reprises, le projet d'une résidence d'études a été discuté avant sa mise en place effective. On nous a toujours donné tous les moyens nécessaires pour développer ce programme et pour l'utiliser comme un pivot permettant de lancer de nouveaux projets (colloques, bourses de voyage, etc...). Par ailleurs, cette idée d'inviter des jeunes artistes avec des doctorants témoignait du désir de recréer une communauté artistique et intellectuelle, de rassembler des esprits créatifs dans un environnement de retraite à la campagne, une idée décidément utopique.

FB : Parlons un peu de l'avenir, avec une transition qui s'impose. Sachant que le Board n'est pas stable, qu'il veut se désinvestir d'opérations très lourdes en matériel et en personnel pour se concentrer sur des programmes de soutien, la question est évidemment : quel est l'avenir probable de ces programmes de soutien, leur pérennité? Avez-vous l'impression que le Board actuel croit profondément à ces programmes éducatifs, ou pas ? Sachant qu'il y a toujours eu en son sein, comme dans n'importe quel board, une tendance pour laquelle le but véritable est de valoriser la collection, et l'art américain en général à travers elle? Corrigez-moi si je dis des bêtises...

SL : Là, je vous arrête. Le cœur de la mission de la Fondation est de faire connaître l'art américain historique en dehors des frontières des Etats-Unis - et aux Etats-Unis. C'était l'idée de Daniel Terra.

FB : Oui, bien sûr - mais d'où vient cette idée, qui est quand même très bizarre, aux yeux des Européens?

VT : Je pense qu'il y a un élément patriotique.

SL : Patriotique, oui, absolument. En fait, il y a cette idée qu'après Pollock l'art américain est devenu l'art international, mais qu'il y avait un art américain avant Pollock. Ce qui est original dans la démarche de Daniel Terra, et que je trouve visionnaire, est qu'il n'a jamais essayé de faire croire que cet art était purement américain - et sa collection le prouve. On avait fait le calcul que quelque chose comme 2/3 de la collection Terra a été produit en dehors des Etats-Unis. Donc, il n'y avait pas du tout la volonté de créer une collection qui essaierait de retracer une origine pure et sans mélange de l'art américain.

VT : Et il était lui-même fils d'immigrés.

SL : Il y avait donc un côté patriotique, mais tout en finesse. Pour lui, cet art américain antérieur à 1940 valait le coup d'être montré, mieux connu, mieux mis en valeur dans les musées américains.

FB : Est-ce qu'il devait être mieux connu parce qu'il était américain ou parce qu'il avait des mérites formels, historiques, etc, trop méconnus? Est-ce que le motif était purement patriotique, ou est-ce qu'il y allait d'un rapport plus général à la peinture ? Je me demande quel était le rapport de Daniel Terra à la peinture, par exemple aux impressionnistes, ou à Homer...

SL : Bingham, par exemple, est l'un des premiers peintres non impressionnistes qu'il a achetés. Daniel Terra n'était pas un théoricien, un penseur, il n'écrivait pas sur ses acquisitions ni sur ses prises de position. C'était quelqu'un d'assez instinctif, et un entrepreneur, aussi.

VT : Ce qui peut également expliquer son intérêt : il voyait une niche.

SL : Oui, c'est très juste. 
FB : Rappelons que Daniel Terra a commencé à acheter de la peinture dans les années 1970..

SL : Ses premières acquisitions n'étaient pas des œuvres américaines mais des paysagistes anglais - avec sa première femme. Puis il y a eu un choix stratégique, plus pensé, partant de l'idée qu'il lui fallait se spécialiser sur l'art américain , qui était alors une zone un peu délaissée par le marché de l'art, et qui lui permettait d'apparaître comme une personnalité importante dans le milieu américain de l'art.

FB : Mais n'était-il pas quand même un latecomer, sur le terrain de l'art américain "historique" ? Il y a eu des grandes collections d'art américain bien avant la collection Terra.

SL : Il y a des collections publiques, oui, mais pas privées, et elles ne s'intéressaient pas forcément aux mêmes moments. Terra a donné une valeur de marché à des mouvements qui étaient très dédaignés - comme le mouvement impressionniste (américain). Il a toujours beaucoup réfléchi à la manière dont il pouvait se différencier des autres fondations, collectionneurs, ou bienfaiteurs culturels américains.

VT : Je pense qu'il y a d'un côté Daniel Terra, de l'autre la Fondation, qui est autre chose, et j'ai toujours été frappée par la tension entre les deux. C'est une fondation qui essayait de se développer, mais qui avait un rapport un peu complexeavec son fondateur.

SL : Je crois que c'est assez classique. Presque toutes les fondations créées par un homme, au moment de sa mort, vivent une crise et une sorte de redéfinition de leur mission.

FB : Venons-en à votre mission éducative, qui va devenir encore plus importante - l'activité principale si je comprends bien - même si vous allez continuer à soutenir des expositions.

SL : Oui, n'oublions pas que dans la distribution des fonds, le soutien aux expositions reste une part beaucoup plus importante, en raison directe du coût de ce genre de projets.

VT : Par ailleurs, le cœur de la Fondation est plus proche du monde des musées que de celui de l'Université.

SL : Mais dès l'origine, la dotation Terra a été séparée en trois fonds distincts, qui donnent une indication très claire sur ses objectifs. Il y avait un petit fonds dédié à l'administration, un fonds assez important dédié aux acquisitions, et un troisième fonds, de loin le plus important, dédié à la mission éducative. On retrouve ce partage dans la définition des guidelines et des objectifs. L'éducation - que ce soit par les expositions, les publications, les projets universitaires - est au cœur de sa mission.

GC : Et alors, quel est le lien entre la mission éducative et la valeur de marché d'une collection privée ? Comment ces deux choses-là étaient-elles articulées ?

SL : La valeur de marché ne joue que dans la mesure où Daniel Terra n'a pas cessé de revendre et d'acheter des œuvres, et que la Fondation a toujours le droit de revendre (deaccession) des œuvres, ce qu'ils ont fait - rarement, et toujours pour racheter d'autres œuvres. Cet aspect est évidemment en contraste avec le monde des musées français, et à cet égard la valeur de marché des œuvres entre en ligne de compte. Cependant, la mission de la collection a toujours été éducative - ce qui explique qu'elle voyage considérablement, qu'elle n'est pas fixe, parce qu'elle sert à monter 
des expositions. Le MAAG l'a fait constamment - a utilisé les œuvres de la collection sous tous les angles possibles et imaginables dans un but éducatif.

VT : Et le musée Terra de Chicago était lui aussi conçu selon ce modèle éducatif. Il y avait énormément de visites guidées, de travail avec des groupes scolaires, et c'est d'ailleurs toujours un programme d'envergure aujourd'hui à Chicago, à travers la formation d'enseignants au niveau primaire et secondaire.

SL : C'est ce qu'ils appellent "K through 12" ("Kindergarten through 12th grade", ce qui correspondrait en France à l'enseignement primaire et secondaire), un programme pédagogique qui vise les enfants à travers la formation des enseignants.

VT : Il y a aussi un soutien à des projets plus ouverts au grand public, visant à montrer comment utiliser ces tableaux pour expliquer l'histoire américaine aux Américains. Ce côté apparaît moins ici parce que, à part les visites au musée, nous ne travaillons pas ici au niveau de l'enseignement primaire et secondaire.

SL : Alors que Daniel Terra était plutôt formaliste, s'intéressait plutôt - d'après ce qu'il a dit - aux tableaux pour leur beauté, il y a eu une légère inflexion de la politique d'acquisitions vers des tableaux permettant de raconter des choses sur l'histoire des Etats-Unis. Récemment a été acheté un tableau de genre peint pendant la guerre de Sécession, et il est question de constituer un petit groupe avec tous les tableaux datant de la guerre, qui permettrait une sorte de narration un peu historique.

FB : Donc les acquisitions continuent?

SL : Oui. Il y a eu, en 2007, l'achat d'un John Marin assez tardif, et ce tableau de Lilly Martin Spencer, The Home of the Red, White and Blue, c. 1867-68:

\section{http://www.terraamericanart.org/collections/code/ emuseum.asp [search: Spencer]}

FB : Revenons au budget actuel, après la fermeture du MAAG. En dehors des acquisitions, les deux principaux postes vont être les expositions et les programmes éducatifs.

SL : Oui, mais il y a aussi les Terra Initiatives, dont on n'a pas beaucoup parlé à l'INHA. On va profiter de la collection et des compétences pour continuer à produire des projets, même s'ils n'auront pas lieu dans nos murs.

\section{GC : Quels types de projets?}

SL : Expositions, colloques, séminaires de recherche, inventaires de l'art américain dans des collections publiques européennes, sous notre label, mais pas seulement comme financeurs, également comme coproducteurs et concepteurs.

VT : Il y aura des projets dont nous sommes les initiateurs et pour lesquels nous prenons part dans le choix des intervenants. Nous avons deux grands projets à Londres, avec un partenariat très prononcé, mais où nous sommes vraiment acteurs. Autre exemple: même si le programme d'un enseignement post-doctoral et de professeurs invités, qui débutera à Paris, Londres et Berlin en automne 2009, prend la forme du mécénat, nous sommes très liés à sa conception et veillerons à son bon déroulement.

SL : Nous serons dans une position un peu plus floue, plus délicate. Il n'y aura pas de projets solitaires, vendus "clés en main"; les projets ne pourront être établis que dans un dialogue avec un partenaire. Mais inversement, rares seront les purs grants, 
parce que notre expertise, celle de Veerle, est et sera très souvent demandée. De même, pour les expositions, nous serons amenés à donner une aide au long de la conception. Il y a par exemple un projet de ce type porté par le Musée Malraux du Havre sur l'histoire des relations artistiques entre Le Havre et New York, comme deux villes portuaires, et nous sommes associés aux différentes étapes du projet. Il va donc devenir difficile de distinguer les grants et les initiatives, et c'est là tout l'intérêt de créer ce bureau européen - car on aurait évidemment pu imaginer faire tout cela depuis Chicago.

FB : Alors justement, comment comprendre ce bureau européen, quel est son avenir ?

VT : C'est important de ne pas être overseas, d'être là, proches, et de faire de ce bureau un lieu de rencontres - avec sa bibliothèque, ses ressources, mais aussi ses personnes, qu'on peut venir voir, qui proposeront l'accueil des enseignants, étudiants, et des moments de débat. Donc, un lieu de ressources beaucoup plus tangible que les headquarters d'une fondation à Chicago.

SL : Ce qui nous apparaît quotidiennement dans notre dialogue avec nos collègues de Chicago est que le malentendu culturel est toujours là, et qu'il faut constamment traduire les choses pour l'autre côté. Expliquer qu'il est plus difficile pour certains musées européens de demander un grant à la Fondation Terra que pour un musée américain... Certains conservateurs ou universitaires américains ont du mal à imaginer que tout le monde ne parle pas anglais, ou que l'histoire de l'art américain n'est pas simplement traduisible mot à mot et exportable sans autre effort. Il manque une conscience que les deux mondes culturels, bien que proches, ont des contextes extrêmement différents, sur lesquels il faut constamment travailler. Cette incompréhension ou cette difficulté à comprendre l'étranger reste réelle - de même que les difficultés qu'ont les Européens à comprendre les logiques américaines restent tout aussi réelles. C'est là que nous nous situons.

FB : Justement, en décidant de vous situer à Paris, vous choisissez la difficulté maximale (en matière d'incompréhension culturelle). Si vous vous situiez en Allemagne ou aux Paysbas, la difficulté ne serait-elle pas moindre?

VT : Oui, mais on repartirait de zéro.

SL : On profite du travail accumulé des années passées. Et c'est justement parce que c'est avec la France que l'incompréhension est à son maximum qu'il faut être là, en s'appuyant sur une dizaine d'années de projets, de liens créés, de ce socle du temps qui passe. L'une des choses que les Européens ont du mal à comprendre, c'est justement, pourquoi changer si vite de direction - alors que pour les Américains, quinze ans, c'est très long, même si rares sont en fait les musées qui ferment aux Etats-Unis.

VT : Je pense quand même qu'il y a dans les rapports franco-américains quelque chose de très vivant. Par contraste, quand je vais à Londres, on me dit, oui, d'accord, on pourrait faire quelque chose sur l'art américain.... mais cela ne constitue qu'une option parmi d'autre, ni vraiment nouvelle, ni provocante, certainement pour des raisons historiques très différentes. Tandis qu'ici, il y a toujours un élément de surprise, de tension qui éveille l'intérêt.

SL : La rivalité est une source d'intérêt qui donne chance au débat.

FB : Sur cette dimension européenne, justement: on a dit, il y a quelque temps, que la Fondation Terra hésitait à continuer à mettre autant d'oeufs dans le panier Europe, et 
qu'elle s'intéressait à d'autres théâtres d'opérations, comme la Chine par exemple, où la Terra a organisé une exposition récemment. Est-ce que vous pensez que l'action en Europe reste une priorité, ou est-ce moins clair?

VT : C'est vraiment une priorité, la base de départ. Mais j'ai souvent cette question à propos des programmes universitaires : ce que vous mettez en place [en Europe], c'est une première étape, mais après y aura-t-il un élargissement vers les pays asiatiques? Mais l'Europe est bien le cœur, le socle.

SL : Oui, et c'est lié au fait que nous nous occupons d'une période historique [XIX ${ }^{\mathrm{e}}$ début $\mathrm{XX}^{\mathrm{e}}$ ] de l'art américain où l'Europe a été le partenaire essentiel. Or, on se rend compte que souvent les projets les plus intéressants et les moins "livrés clés en main " en Europe sont ceux dans lesquels les Européens arrivent à trouver comment leur art s'est placé en relation avec l'art américain. Un projet en Allemagne, par exemple, concernerait justement cette partie de l'école américaine qui a eu des relations fortes avec l'art allemand. Des liens de ce type avec l'Asie peuvent être faits, mais ils sont beaucoup plus ténus. D'autre part, tout simplement, ce socle historique institutionnel n'existe pas pour l'instant en Asie, même si c'est bien l'envie du Board de s'adresser... worldwide. En Asie, il y a déjà des projets. Il y a eu cette exposition dont vous parliez, co-produite avec le Guggenheim. Il y a aussi le petit projet consistant à faire voyager une autre version de l'exposition "Giverny colonie impressionniste" au Japon, et nous avons été intéressés par ce projet parce que c'est une autre manière de rencontrer le monde des musées japonais - autrement que par ces sociétés qui vendent des expositions clés en main. Il s'agirait là de rencontrer directement directeurs et conservateurs de musées japonais, et par conséquent de commencer à comprendre comment le pays fonctionne sur le plan culturel, et quel pourrait être l'intérêt pour d'autres projets ultérieurs sur l'art américain, avec des universités ou avec d'autres musées.

GC : On voulait aussi vous demander s'il y a un "message" que vous aimeriez faire passer à nos collègues universitaires - s'il y en a un ? Y a-t-il quelque chose à attendre en particulier de la transformation de la structure en cours?

FB : Je rappelle que Transatlantica est une publication de I'AFEA. L'AFEA n'a pas eu en tant que telle de rapports avec la Fondation Terra, en revanche un certain nombre d'individus, de centres de recherches et d'universités liés à l'AFEA en ont eu - plutôt heureux en général, je crois. Donc, naturellement, les gens se demandent ce qu'ils peuvent espérer pour l'avenir. [RIRES] Vous avez répondu, en général, lors de la présentation à I'INHA de votre nouveau centre, en présentant les différentes aides que vous allez proposer; mais souhaitez-vous ajouter quelque chose sur ce chapitre?

VT : Clairement, nous sommes à un moment où on pourra appuyer beaucoup plus de projets externes. Si vous avez un projet, venez nous voir pour en parler. La nouvelle organisation a un côté plus ouvert, moins formel. Organiser des manifestations à l'intérieur d'un musée est quelque chose de très lourd; là, j'espère que ça va bouger un peu, y compris avec des "petits" projets.

GC : Des projets de colloques, de journées d'études, de publications? Des choses en particulier, ou tout projet lié aux arts américains?

VT : Tout projet.

SL : De plus, la fondation aura ce lieu, au 29 rue des Pyramides, où on pourra trouver des documents, des livres, ainsi que des réponses à des questions du type "Quels sont les spécialistes de tel sujet aux Etats-Unis, en Angleterre, en Allemagne ?" - même si on n'a pas la réponse, on peut proposer des pistes pour la chercher. Donc c'est 
vraiment un lieu d'échange d'information et d'entraide sur tout projet sur l'art américain.

FB : Vous avez mentionné, lors de la séance à l'INHA, un site web. Ce sera le site de la Terra ou un autre site?

VT : Toutes les informations sur le centre et ses activités pourront être trouvées sur le site WWW.terraamericanart.org.

FB : Ce site, qui contient déjà beaucoup de choses, est donc appelé à contenir plus d'informations du type de celles que vous évoquiez, par exemple sur les spécialistes de tel ou tel sujet?

VT : Oui - pas des bibliographies, ce n'est pas notre rôle, mais des axes, des relais d'information.

SL : Veerle a aussi un projet ambitieux. Il concerne le groupe des personnes qui travaillent sur l'art américain historique avec lesquelles on a été en contact à travers la TSR, les travel grants, les projets d'exposition, et ce groupe s'élargit, même s'il reste encore de taille humaine (ce ne sont pas des milliers de gens) ; notre centre pourrait être un lieu de mise en réseau de toutes ces personnes, où qu'elles soient.

FB: Vous avez dit que vous alliez souscrire des abonnements à des revues électroniques et des bases de données. Ces ressources seront consultables depuis la bibliothèque?

VT : Oui, c'est ça.

FB : C'est important, car ce qui est disponible actuellement varie beaucoup d'une université à l'autre. L'INHA a bien sûr des ressources importantes, en revanche les universités individuelles n'ont pas forcément beaucoup de ressources électroniques sur l'art américain, même quand, comme la mienne, elles ont fait un effort considérable en matière d'abonnements électroniques aux grands portails.

SL : Oui, c'est important. Et dans ce domaine, nous sommes totalement ouverts aux suggestions, surtout que nous n'avons pas encore commencé. Il serait important de savoir, quant aux bases de données, ce qui manque en France et que la Fondation pourrait mettre à la disposition du public du centre.

FB : Et la TSR ? Elle continue pour le moment, mais connaît-on son avenir au-delà de deux ans?

VT : Une grande évaluation va avoir lieu sur ce point, avec la proposition de continuer car c'est devenu un programme phare dans le champ de l'étude de l'art américain et les échanges culturels aux Etats-Unis et ici. Mais de toute façon, la TSR a toujours été programmée de deux ans en deux ans. Donc, ça continue et cette année, on va voter sur 2010-2011. Je vais mettre l'accent sur les avantages du site givernois, lieu propice à la vie en groupe, ponctuée d'échanges, espacée par des séances de travail individuel, qui ne sont pas possibles à Paris. Ce qui ne veut pas dire qu'il faut garder éternellement le même format. Au bout de neuf ans, peut-être faut-il repenser certaines choses.

SL : Sans compter que la résidence aura pour voisin le Musée des impressionnismes. Le fait qu'il y ait un centre à Paris, et que le Musée de Giverny ne sera plus un musée d'art américain mais un Musée des impressionnismes, va naturellement amener à réviser le format de la TSR, avec peut-être un mélange d'éléments parisiens et givernois. Il s'agira peut-être d'utiliser la meilleure part des deux lieux, car nous gardons les maisons de Giverny. 
GC : Pouvez-vous revenir sur le projet d'aide à la publication présenté par Francesca Rose à I'INHA?

SL: Oui, un de nos projets concerne des grants sur les publications. Une des directions sur lesquelles nous nous sommes un peu penchées est un projet de textbook, un livre relativement grand public, dans le genre de celui de Frances Pohl, par exemple (A Social History of American Art, 2002). Là, on s'adresse à un public de niveau licence, voire plus large encore. On se pose plusieurs questions. L'une des choses que Francesca Rose aimerait faire est d'interroger des enseignants universitaires sur l'utilité - ou l'inutilité - d'un tel outil. Avant de le faire, on voudrait savoir si un tel livre correspond à un besoin, ou non. Il ne s'agirait pas forcément d'une traduction pure et simple d'un ouvrage américain existant, mais peut-être d'une commande à un ou plusieurs auteur(s), ou d'une réécriture plus ou moins approfondie.

FB : L'une des questions qui se pose à ce sujet est la suivante: est-ce que ce qui vous intéresse est de faire connaître des textbooks américains en Europe non seulement pour les matières traitées mais aussi à cause de l'approche qui est la leur et qui, en matière d'histoire sociale de l'art et a fortiori de visual culture, est très peu connu et très peu pratiqué en Europe, ou du moins en France (car en Angleterre et même en Allemagne cette approche est beaucoup plus répandue)? Ou bien, et il me semble qu'il y a les deux perspectives, seriez-vous intéressés, en sens inverse, à recueillir le travail et le point de vue d'universitaires européens ou français sur le champ "art américain", auquel cas la traduction pourrait se faire dans l'autre sens? II est sûr, à mon avis, qu'il y a besoin en France de ce type d'outil, mais je pense pour ma part que l'on pourrait coupler la découverte de ces matières peu connues en France avec un renouvellement méthodologique inspiré par tout ce qui va de l'histoire sociale de l'art à la visual culture.

VT: La Terra est plutôt associée à la tradition "beaux-arts", mais en même temps, dans tous nos projets nous n'avons jamais exclu l'approche visual culture. Et l'approche sociale, étant au cœur de l'étude de l'art américain, a souvent été mise en avant dans nos colloques et journées d'études. Le colloque 'Narratives about American art' qui s'est tenu au John F. Kennedy à Berlin il y a deux ans, avait une entrée explicitement historiographique. A l'avenir, nous voudrions solliciter ce genre de réflexion encore davantage. La publication de textes par des chercheurs européens est évidemment un axe qui nous intéresse beaucoup. Il permettra de créer un dialogue dans l'autre sens et de briser l'isolement dans lequel se trouve la recherche purement américaine.

INDEX

Thèmes : Trans'Arts

\section{AUTEURS}

\section{FRANÇOIS BRUNET}

Université Paris-Diderot Paris-7 


\section{GÉRALDINE CHOUARD}

Université Paris-Dauphine 